\title{
GROUP THEORETIC REMARKS ON RIESZ SYSTEMS ON BALLS ${ }^{1}$
}

\author{
A. KORÁNYI AND S. VÁGI
}

\begin{abstract}
The space of boundary values of Riesz systems on the unit ball of $\mathbf{R}^{n}$ is decomposed under the action of the special orthogonal group $S O(n)$. The corresponding irreducible representations are explicitly determined. Two applications of this result are given.
\end{abstract}

Introduction. In [3 and 4] we considered $\mathbf{R}^{n}$-valued functions on the unit sphere $S^{n-1}$ of $\mathbf{R}^{n}$ and discussed the generalization of the results of Marcel Riesz on the conjugate function to this setting. This was done with the aid of a theory of singular integral operators developed in [3]. It is well known that, just as in the case of the circle and the real line, the Fourier transform carries singular integral operators of the type considered in [3] into multiplier operators. In the present paper we shall describe concretely the Fourier analysis of $\mathbf{R}^{n}$-valued functions on $S^{n-1}$, and we shall identify the multiplier corresponding to the Riesz transform. In $\S 2$ we shall apply our Fourier analysis to prove that, in analogy with the complex analytic case, the restriction of the generalized Cauchy-Riemann equations to $S^{n-1}$ characterizes the tangential parts of the boundary values of Riesz systems. As it will turn out from our discussion, this is really a result about harmonic forms, and is essentially contained in [2]; our proof is an analog of the classical method of solving the Dirichlet problem for the ball by means of an expansion into spherical hamonics.

Throughout this paper we restrict our attention to the case $n \geq 5$. The cases $n=3,4$ require some modifications corresponding to modifications occurring in the Branching Theorem; the case $n=2$ is the classical case of the unit circle.

1. Decomposition of a representation. We write $L=L^{2}\left(S^{n-1}, \mathbf{R}^{n}\right)$ and we consider the representation $T$ of the special orthogonal group $S O(n)$ given for $g \in$ $S O(n), f \in L$, and $x^{\prime} \in S^{n-1}$ by

$$
\left(T_{g} f\right)\left(x^{\prime}\right)=g f\left(g^{-1} x^{\prime}\right)
$$

If we interpret the elements of $L$ as sections of the trivial vector bundle $S^{n-1} \times \mathbf{R}^{n}$ with $S O(n)$ acting in the natural way on both factors, then $T$ is just the action of $S O(n)$ on the sections. Our first goal is to decompose $T$ into irreducible constituents.

We define, using the dot to denote the standard inner product on $\mathbf{R}^{n}$,

$$
\begin{aligned}
& L_{\mathrm{Tan}}=\left\{f \in L \mid f\left(x^{\prime}\right) \cdot x^{\prime}=0, x^{\prime} \in S^{n-1}\right\}, \\
& L_{\mathrm{Nor}}=\left\{f \in L \mid f\left(x^{\prime}\right)=\varphi\left(x^{\prime}\right) x^{\prime}, \varphi \in L^{2}\left(S^{n-1}, \mathbf{R}\right)\right\} .
\end{aligned}
$$

Received by the editors February 10, 1981 and, in revised form, September 14, 1981.

1980 Mathematics Subject Classification. Primary 43A85; Secondary 42B15, 42B20.

${ }^{1}$ Research partially supported by NSF grants.

(C) 1982 American Mathematical Society 0002-9939/81/0000-0132/802.50 
These spaces are $S O(n)$-invariant, and $L$ is the orthogonal direct sum

$$
L=L_{\mathrm{Tan}} \oplus L_{\mathrm{Nor}},
$$

as can be seen by decomposing $f \in L$ as $f=f_{\text {Tan }}+f_{\text {Nor }}$, where $f_{\text {Nor }}\left(x^{\prime}\right)=$ $f\left(x^{\prime}\right) \cdot x^{\prime}$.

If we think of $L$ as a space of vector-valued functions (or "vector-fields"), $L_{\text {Tan }}$ consists of the tangential, $L_{\text {Nor }}$ of the normal fields. Actually, it is better to think of them as covector-valued functions; it is, in fact, easy to verify the $L_{\text {Tan }}$ is isomorphic as a homogeneous space with the cotangent bundle of $S^{n-1}$.

To proceed with the decomposition of $L$ we introduce some notation. Let $D^{r, 0}$ resp. $D^{r, 1}$ denote the irreducible representation of $S O(n)$ whose highest weight (cf. $[1])$ is $(r, 0, \ldots, 0)$ resp. $(r, 1,0, \ldots, 0)$. Let $\mathcal{H}_{r}$ denote the space of homogeneous harmonic polynomials of degree $r$ on $\mathbf{R}^{n}$. Then, as is well known, $S O(n)$ operates on $\mathcal{K}_{r}$ (and also on the restrictions of $\mathcal{H}_{r}$ to $S^{n-1}$ ) by the representation $D^{r, 0}$.

PROPOSITION 1.1. One has a unique orthogonal decomposition

$$
L_{\mathrm{Nor}}=\bigoplus_{r \geq 0} L_{\mathrm{Nor}}^{r, 0}
$$

where $S O(n)$ acts on $L_{\text {Nor }}^{r, 0}$ by the irreducible representation $D^{r, 0}$.

Proof. The map $\varphi \mapsto A \varphi$, where $(A \varphi)\left(x^{\prime}\right)=\varphi\left(x^{\prime}\right) x^{\prime}$, is an $S O(n)$-equivariant isomorphism of $L^{2}\left(S^{n-1}, \mathbf{R}\right)$ onto $L_{\mathrm{Nor}}$. The assertion follows from the classical decomposition of $L^{2}\left(S^{n-1}, \mathbf{R}\right)$ into spherical harmonics. In addition one has the explicit description

$$
L_{\text {Nor }}^{r, 0}=\left\{\varphi\left(x^{\prime}\right) x^{\prime} \mid \varphi \in \not_{r}\right\}
$$

PROPOSITION 1.2. One has a unique orthogonal decomposition

$$
L_{\text {Tan }}=\bigoplus_{r \geq 1}\left(L_{\text {Tan }}^{r, 0} \oplus L_{\text {Tan }}^{r, 1}\right)
$$

where $S O(n)$ acts on $L_{\text {Tan }}^{r, i}$ by the irreducible representation $D^{r, i}(i=0,1)$.

ProOF. Let $e_{n}=(0, \ldots, 0,1)$. The restriction of $T$ to $L_{\mathrm{Tan}}$ is just the representation induced by the canonical representation on $\mathrm{R}^{n-1}$ of $S O(n-1)$, the stabilizer of $e_{n}$. In fact, writing $\tilde{f}(g)=\left(T_{g} f\right)\left(e_{n}\right)$, one verifies at once that $\tilde{f}$ is a function from $S O(n)$ to $\mathbf{R}^{n-1}$ (the tangent space to $S^{n-1}$ at $e_{n}$ ) and, for $g \in S O(n), k \in$ $S O(n-1)$ we have $\tilde{f}(k g)=k \cdot \tilde{f}(g)$ and $\left(T_{g} f\right) \sim\left(g^{\prime}\right)=\tilde{f}\left(g^{\prime} g\right)$, proving our assertion. Now the Frobenius reciprocity theorem [6] implies that the irreducible representations occurring in $L_{\text {Tan }}$ are those whose restriction to $S O(n-1)$ contains the canonical representations of $S O(n-1)$ (which is of type $D^{1,0}$ ). By the Branching Theorem [1, pp. 248-251] this means exactly those whose highest weight $\left(m_{1}, \ldots, m_{p}\right)$ satisfies

$$
m_{1} \geq 1 \geq m_{2} \geq 0 \geq m_{3} \geq \ldots
$$

i.e. the representations $D^{r, 0}$ and $D^{r, 1}$ with multiplicity one for each $r \geq 1$.

We are now going to consider the subspace $H$ of $L$ formed by the boundary values (in the $L^{2}$ sense) of gradients $\nabla \varphi=\left(D_{1} \varphi, \ldots, D_{n} \varphi\right)$ of harmonic functions, where we used the standard notation $D_{j} \varphi=\partial \varphi / \partial x_{j}$. This is the space denoted by $H^{2}\left(S^{n-1}\right)$ in [4]. 
Since $\nabla$ intertwines the action of $S O(n)$ on functions and on $L, H$ is an invariant subspace of $L$ and we have

$$
H=\bigoplus_{r \geq 1} H^{r, 0}
$$

with

$$
H^{r, 0}=\left\{\nabla \varphi \mid \varphi \in \mathfrak{H}_{r}\right\}
$$

transforming under $T$ by the representation $D^{r, 0}$. The space $H^{r, 0}$ is contained in $L_{\text {Nor }}^{r, 0} \oplus L_{\text {Tan }}^{r, 0}$ but is itself (cf. (1.8) and (1.9) below) neither normal nor tangential.

Let $L_{\mathrm{Nor}, 0}$ be the subspace of $L_{\mathrm{Nor}}$ made up of elements $\varphi\left(x^{\prime}\right) x^{\prime}$ with $\int \varphi=0$, i.e. the sum (1.3) without the term $r=0$. We write $\operatorname{pr}_{\mathrm{Tan}}$ and $\mathrm{pr}_{\text {Nor }}$ for the projections corresponding to the decomposition (1.2) of $L$. The Riesz Transform $R$ is defined in [3] as the mapping of $L_{\mathrm{Nor}, 0}$ into $L_{\mathrm{Tan}}$ carrying $\operatorname{pr}_{\mathrm{Nor}} \nabla \varphi$ to $\operatorname{pr}_{\mathrm{Tan}} \nabla \varphi$ for all $\varphi \in \mathcal{H}_{r}(r \geq 1)$. The operator $R$ is clearly an $S O(n)$-equivariant map. The following proposition describes the "multiplier" corresponding to $R$.

Proposition 1.3. For each $r \geq 1$, the restriction of $R$ to $L_{\text {Nor }}^{r, 0}$ is

$$
\left(\frac{r+n-2}{r}\right)^{1 / 2} V_{r}
$$

where $V_{r}$ is an $S O(n)$-equivariant isometry of $L_{\text {Nor }}^{r, 0}$ onto $L_{\text {Tan }}^{r, 0}$.

ProOF. By the computations in $[4$, p. 186] we have

$$
\|\nabla \varphi\|^{2}=(n+2 r-2) r \int_{S^{n-1}}|\varphi|^{2} .
$$

By Euler's identity, $x \cdot \nabla \varphi(x)=r \varphi(x)$, hence $\left(\operatorname{pr}_{\text {Nor }} \nabla \varphi\right)\left(x^{\prime}\right)=r \varphi\left(x^{\prime}\right) x^{\prime}$. Therefore,

$$
\left\|\operatorname{pr}_{\text {Nor }} \nabla \varphi\right\|^{2}=r^{2} \int_{S^{n-1}}|\varphi|^{2}
$$

and, by the theorem of Pythagoras,

$$
\left\|\mathrm{pr}_{\mathrm{Tan}} \nabla \varphi\right\|^{2}=(n+r-2) r \int_{S^{n-1}}|\varphi|^{2} .
$$

REMARK. The operator $P$ of [4] projects in a group-equivariant way each $L_{\text {Tan }}^{r, 0} \oplus L_{\text {Nor }}^{r, 0}$ onto $H^{r, 0}$, and maps each $L_{\text {Tan }}^{r, 1}$ to zero.

The next proposition will make the decomposition of $L$ more explicit. Consider $\mathcal{H}_{r} \otimes \mathbf{R}^{n}$, i.e. the $n$-tuples of elements of $\mathfrak{H}_{r}$. This space (more exactly the restriction of its elements to $S^{n-1}$ ) is contained in $L$, and since the spherical harmonics span $L^{2}\left(S^{n-1}, \mathbf{R}\right)$ we have the orthogonal decomposition

$$
L=\bigoplus_{r \geq 0} H_{r} \otimes R^{n}
$$

Here $S O(n)$ acts on the $r$ th summand by $D^{r, 0} \otimes D^{r, 1}$; it is known (cf. [5]) that this tensor product equals $D^{r, 0} \oplus D^{r+1,0} \oplus D^{r-1,0}$. Since $D^{r, 1}$ occurs in $L$ only once, the subspace of type $D^{r, 1}$ must be $L_{\text {Tan }}^{r, 1}$. Since the components of the elements of $H^{r+1,0}$ are harmonic polynomials of degree $r$, we have $H^{r+1,0} \subset \varkappa_{r} \otimes R^{n}$, so this must be the subspace of type $D^{r+1,0}$. Denoting the subspace of type $D^{r-1,0}$ by $M^{r-1,0}$ we have then

$$
\mathcal{K}_{r} \otimes \mathbf{R}^{n}=L_{\mathrm{Tan}}^{r, 1} \oplus H^{r+1,0} \oplus M^{r-1,0} .
$$


PROPOSITION 1.4. For each $r \geq 1$ there is another orthogonal decomposition

$$
L_{\text {Tan }}^{r, 0} \oplus L_{\text {Nor }}^{r, 0}=H^{r, 0} \oplus M^{r, 0} .
$$

Furthermore,

$$
M^{r, 0}=\left\{|x|^{2} \nabla \varphi(x)-(n+2(r-1)) \varphi(x) x \mid \varphi \in \mathcal{H}_{r}\right\} .
$$

Proof. By (1.3), (1.5) and (1.11), $M^{r, 0}$ is the kernel of the projection $P$ on $L_{\text {Tan }}^{r, 0} \oplus L_{\text {Nor }}^{r, 0}$. This implies (1.12). We can determine $M^{r, 0}$ explicitly as follows. For $\varphi \in \not{H}_{r}$ we have $\varphi(x) x=h_{r+1}(x)+|x|^{2} h_{r-1}(x)+|x|^{4} h_{r-3}(x)+\ldots$, with $h_{i} \in \mathcal{H}_{i} \otimes \mathbf{R}^{n}$. Since $\varphi(x) x$ is of type $D^{r, 0}$, by (1.10) and (1.11) it follows that $h_{r+1} \in M^{r, 0}, h_{r-1} \in H^{r, 0}$ and $h_{i}=0$ for $i<r-1$. So $h_{r-1}$ is the orthogonal projection of $\varphi(x) x$ onto $H^{r, 0}$, and is found by maximizing $(\varphi(x) x \mid \nabla \psi) /\|\nabla \psi\|$ as $\psi$ ranges over $\mathfrak{H}_{r}$. The Euler identity, (1.7) and the Schwarz inequality then show that $h_{r-1}=(n+2 r-2)^{-1} \nabla \varphi$. This proves (1.13). Of course, one could also verify directly that the right-hand side of (1.13) is made up of harmonic polynomials of degree $r+1$, and is of type $D^{r, 0}$; this would suffice to prove that it is equal to $M^{r, 0}$.

REMARK. It may be of interest to point out the connections of the preceding with Levine's work [5]. Levine considers the harmonic analysis of the cosphere bundle of $S^{n-1}$ concretely realized as

$$
C S=\left\{(x, \xi)\left|x, \xi \in \mathbf{R}^{n},\right| x|=| \xi \mid=1, x \cdot \xi=0\right\} .
$$

Given $f \in L_{\mathrm{Tan}}$, we can define a function $\tilde{f}$ on $C S$ by $f(x, \xi)=f(x) \cdot \xi$. This gives a bijection of $L_{\mathrm{Tan}}$ onto the subspace of $L^{2}(C S)$ spanned by the functions linear in $\xi$; it is also easy to check that $\left(T_{g} f\right) \sim(x, \xi)=\tilde{f}\left(g^{-1} x, g^{-1} \xi\right)$. With the aid of these observations one can derive (1.5) from Theorems 1,2 and 3 of [5].

2. The tangential Cauchy-Riemann equations. For functions $f$ with values in a vector space $V$ on a manifold $M$ the first order linear differential operators are the same as the $V^{*}$-valued vector fields, i.e. for each $x \in M$ an element $D_{x}$ in $M_{x} \otimes V^{*}$. If $D_{x}=X \otimes e$, then $D_{x} f=X\langle e, f\rangle$. A system $S$ of first order operators is a $C^{\infty}(M)$-module of $V^{*}$-valued vector fields. If $N$ is a submanifold of $M$, the restriction $S_{N}$ of the system $S$ is given by those $D \in S$ for which $D_{x} \in N_{x} \otimes V^{*}$ for all $x \in N$.

The generalized Cauchy-Riemann system (cf. [7]) on $\mathbf{R}^{n}$ (in the following: C-R system) is obtained by taking $V=\mathbf{R}^{n}$, identifying $\mathbf{R}^{n}$ with its dual, and taking the system generated by

$$
\begin{aligned}
D^{0} & =\sum_{1 \leq i \leq n} D_{i} \otimes e_{i}, \\
D^{j k} & =D_{k} \otimes e_{j}-D_{j} \otimes e_{k} \quad(1 \leq j<k \leq n),
\end{aligned}
$$

where $\left\{e_{i}\right\}$ is the natural basis of $\mathbf{R}^{n}$. Suppose that $N$ is a hypersurface in $\mathbf{R}^{n}$ and that at some $x=x_{0} \in N$ the tangent space is spanned by $e_{1}, \ldots, e_{n-1}$. To compute the restriction of the C-R system we write $D_{x}=a_{0} D^{0}+\sum a_{j k} D^{j k}$, and verify that $D_{x} \in N_{x}$ amounts to $a_{0}=a_{j n}=0$ for all $1 \leq j \leq n-1$.

In the case of $N=S^{n-1}$ and $x_{0}=e_{n}$ the last condition means that

$$
\left.\left(D_{k} f_{j}-D_{j} f_{k}\right)\right|_{e_{n}}=0 \quad(1 \leq j<k \leq n-1) .
$$


If, for $f \in L$, we define $\theta_{f}$ as the form on $S^{n-1}$ corresponding to $\operatorname{pr}_{\operatorname{Tan}} f$ and we define the operator $D$ by

$$
D f=d \theta_{f}
$$

(in the sense of distributions), we see at once that (2.1) is equivalent to $(D f)\left(e_{n}\right)=$ 0 , and by $S O(n)$-invariance it follows that $f$ satisfies the restricted C-R system if and only if

$$
D f=0 .
$$

(We might note that the necessity of (2.2) also follows by observing that the C-R system for a Riesz system $F$ is equivalent with $d \theta_{F}=d^{*} \theta_{F}=0$, where $\theta_{F}$ is the form corresponding to $F$; if $f$ is the restriction of $F$ to a submanifold, then $\theta_{f}$ is just the pull-back of $\theta_{F}$ under the identity map, so $d \theta_{f}=0$.) Since it is satisfied by all normal fields, the restricted C-R system cannot, as in the complex case, characterize the elements of $H$. However we have the following result.

Proposition 2.1. An element $f$ on $L_{\mathrm{Tan}}$ is the tangential projection of some element in $H$ if and only if $D f=0$ in the sense of distributions.

Proof. Suppose that $f$ is the tangential part of the boundary values of a Riesz system $F$. Writing $F_{r}(x)=F(r x)(0<r<1)$ it is clear that $F_{r}$ is still a Riesz system. So its restriction to $S^{n-1}$ satisfies (2.2). Since $D$ annihilates all normal fields, so does its tangential projection. Letting $r$ tend to 1 , it follows that $D f=0$.

To prove the converse, we assume $D f=0$, and write $f=\sum f^{r, 0}+\sum f^{r, 1}$ corresponding to the decomposition (1.5). $D$ commutes with the action of $S O(n)$ since the maps $f \mapsto \theta_{f}$ and $\theta_{f} \mapsto d \theta_{f}$ do. Therefore $D f^{r, 1}=0$ for all $r$. From $\S 1$ we know that

$$
\operatorname{pr}_{\mathrm{Tan}} H^{r, 0}=L_{\text {Tan }}^{r, 0}
$$

so the proposition will be proved if we show that $f^{r, 1}=0$ for all $r$. By Schur's lemma, $D$ restricted to $L_{\text {Tan }}^{r, 1}$ is either zero or an isomorphism; it will therefore be sufficient to prove that it cannot be zero. Now notice that $D$ annihilates each $H^{r}$ and $M^{r}$, by (1.12) and (2.3). Therefore, by (1.11), it will suffice to find an element of $H_{r} \otimes \mathbf{R}^{n}$ on which $D$ does not vanish.

Let $g=\left(g_{1}, \ldots, g_{n}\right)$ be defined by $g_{1}(x)=\left(x_{2}+i x_{n}\right)^{r}$, and $g_{2}=\cdots=g_{n}=0$. Then

$$
\left.\left(D_{2} g_{1}-D_{1} g_{2}\right)\right|_{e_{n}}=r i^{r-1} \text {. }
$$

Depending on the parity of $r, D(\operatorname{Re} g)$ or $D(\operatorname{Im} g)$ is different from zero. Since $\operatorname{Re} g$ and $\operatorname{Im} g$ are elements of $H_{r} \otimes \mathbf{R}^{n}$ this concludes the proof.

This proof can also be extended to the case of higher degree forms.

\section{REFERENCES}

1. H. Boerner, Darstellungen von Gruppen, 2nd ed., Springer, Berlin, 1976.

2. W. V. D. Hodge, A Dirichlet problem for harmonic functionals with applications to analytic varieties, Proc. London Math. Soc. 36 (1934), 257-303.

3. A. Korányi and S. Vági, Singular integrals on homogeneous spaces and some problems of classical analysis, Ann. Scuola Norm. Sup. Pisa 25 (1971), 576-648.

4. _ Cauchy-Szegö integrals for systems of harmonic functions, Ann. Scuola Norm. Sup. Pisa 26 (1972), 181-196. 
5. D. A. Levine, Systems of singular integral operators on spheres, Trans. Amer. Math. Soc. 144 (1969), 493-522.

6. L. Pukánszky, Leçons sur les représentations des groupes, Dunod, Paris, 1967.

7. E. M. Stein and G. Weiss, Introduction to Fourier analysis on Euclidean spaces, Princeton Univ. Press, Princeton, N. J., 1971.

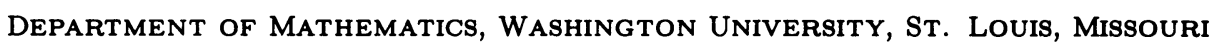
63130

I. R. M. A. Université de Strasbourg, Strasbourg, France

Department of Mathematics, DePaul University, Chicago, Illinois 60614 